\title{
Familial 4;18 chromosome translocation resulting in trisomy $4 p$ and monosomy 18p: affected individuals with discordant phenotype
}

\author{
Deborah R. Berman ${ }^{1 *}$, Carrie A. Couyoumjian ${ }^{1}$, Marjorie C. Treadwell ${ }^{1}$ and Mason Barr Jr$^{2}$ \\ ${ }^{1}$ Perinatal Assessment Center, Division of Maternal-Fetal Medicine, Department of Obstetrics and Gynecology, University of \\ Michigan, Ann Arbor, Michigan, USA \\ ${ }^{2}$ Teratology Unit, Department of Pediatrics, Pathology and Obstetrics and Gynecology, University of Michigan, Ann Arbor, \\ Michigan, USA
}

KEY WORDS: unbalanced chromosome rearrangement; congenital anomalies; proboscis; fetal ultrasound; fetal imaging; general cytogenetics; prenatal cytogenetics; genetic counseling

Balanced chromosome translocations in either parent increase the risk of recurrent miscarriage, unbalanced chromosome rearrangements, congenital malformations, and mental retardation in liveborn offspring. Chromosome aberrations account for at least $50 \%$ of fetal losses prior to 15 weeks' estimated gestation (EGA). Of these, $60 \%$ occur secondary to autosomal trisomies (Gardner and Sutherland, 2004). Prenatal detection of parental chromosome rearrangements provides an opportunity to plan prenatal screening and diagnostic procedures, including chorionic villus sampling (CVS), amniocentesis, early sonographic evaluation, and pre-implantation genetic diagnosis and treatment.

We report a familial chromosome translocation involving chromosomes 4 and 18 resulting in multigenerational history of recurrent miscarriage and two offspring with unbalanced chromosome rearrangements and phenotypic and neurodevelopmental sequelae. This case report describes, for the first time, a translocation that resulted in the combination of monosomy $18 \mathrm{p}$ and trisomy $4 \mathrm{p}$.

The consultand, III-2 (Table 1), a 32-year-old gravida 1 , presented at $102 / 7$ weeks EGA requesting CVS due to personal and family history of a known chromosome translocation. CVS was not technically possible. Transvaginal fetal ultrasonography showed mid-gut umbilical herniation considered a normal variant given gestational age. Encephalocele was suspected. A protuberant structure from the front of the fetal head consistent with a proboscis was identified. Bilateral clubfeet were suspected.

After counseling, given her balanced translocation and ultrasound findings, III-2 elected voluntary pregnancy termination with concomitant fetal karyotype analysis and autopsy. Dilation and evacuation were performed

\footnotetext{
*Correspondence to: Deborah R. Berman, Department of Obstetrics and Gynecology/Maternal-Fetal Medicine, Room F4811 Mott, 1500 E. Medical Center Dr., Ann Arbor, MI 48109-0264, USA. E-mail: debster@med.umich.edu
}

with resultant fetal fragmentation. Karyotype analysis demonstrated: 46,XX,der(18)t(4;18)(p12;p11.2)mat; band resolution 400. Inspection of fetal remnants confirmed the presence of a proboscis. Early gestational age and fragmented fetal tissue limited examination. Limbs were all pentadactyl without deformity. Holoprosencephaly was inferred from the presence of a proboscis. Due to lack of brain morphology preservation, encephalocele was not confirmed.

III-1, sister of III-2, was a 35-year-old gravida 6, para 2. Her first five pregnancies, with a previous partner, resulted in three first-trimester spontaneous abortions, one ectopic pregnancy, and a healthy daughter. Due to recurrent miscarriages, prior to her fifth pregnancy, peripheral blood karyotype analysis was performed demonstrating balanced chromosome translocation: 46,XX,t(4;18)(p12;p11.2), band resolution 625 . During this pregnancy, due to her balanced translocation, she underwent an amniocentesis. Karyotype analysis indicated a female fetus with a balanced translocation. The pregnancy resulted in the birth of a daughter without physical or developmental concerns, IV-1.

Seven years later, III-1 presented at 20 weeks EGA with a pregnancy conceived with a new partner. Visualization of a single umbilical artery on outside ultrasound, abnormal maternal serum screen significant for estriol $=0.32 \mathrm{MoM}$, and her translocation carrier status prompted referral. At our institution, ultrasound dating the pregnancy was reviewed and estimated delivery date reassigned. By this revision, the maternal serum screen did not indicate increased risk for Down syndrome, trisomy 18, neural tube defects, or Smith-Lemli-Opitz syndrome. A single umbilical artery was confirmed and bilateral hydronephrosis identified. Amniocentesis was performed, with the following karyotype: 46,XYder(18)t(4;18)(p11;p11.1), band resolution 400 , a male fetus with trisomy $4 \mathrm{p}$ and monosomy $18 \mathrm{p}$. The patient was counseled regarding increased risk of physical anomalies and adverse neurodevelopmental 
Table 1 -Pedigree depicting familial $(4 ; 18)$ chromosome translocation in both balanced and unbalanced individuals affecting multiple generations

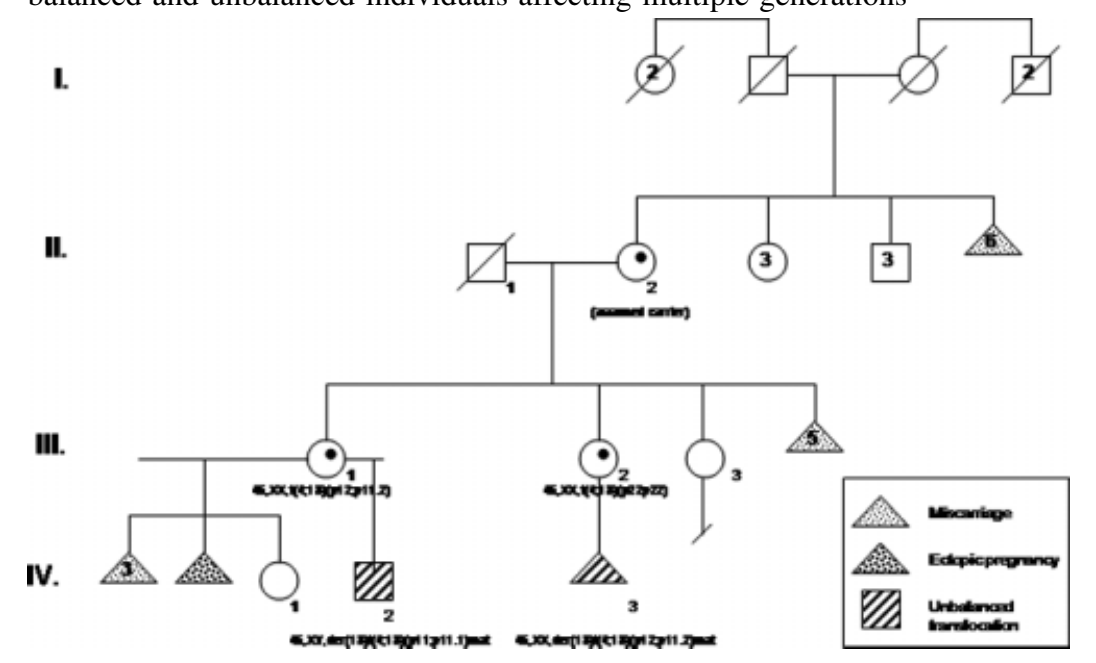

outcomes associated with unbalanced chromosome rearrangements.

Ultrasonography at 22 weeks' EGA confirmed initial ultrasound findings and showed borderline ventriculomegaly with atria of the lateral ventricles measuring $10 \mathrm{~mm}$. Fetal echocardiogram performed at 23 weeks' EGA showed a dilated right ventricle and suspicion of aortic stenosis, aortic coarctation, and ventricular septal defect. Serial ultrasounds documented appropriate interval growth. A liveborn male infant (IV-2) weighing $2394 \mathrm{~g}$ was delivered via repeat Cesarean section at 38 2/7 weeks EGA.

Due to respiratory distress at birth, IV-2 was intubated, received treatment for presumed pneumonia, and was subsequently extubated. During his initial 15-day NICU stay, he required supportive measures for pulmonary and feeding complications. At 2 months, he underwent aortic coarctation repair. Two weeks later, due to respiratory failure, subglottic stenosis, and multiple recurrent intubations, a temporary tracheostomy was performed. Bronchomalacia and tracheomalacia were identified during intraoperative laryngoscopy. At 8 months, evaluation by pediatric genetics demonstrated weight, height, and head circumference below fifth percentile. His ears were normally formed and positioned, and he demonstrated overlapping right foot second over first toe and overlapping first over second toe on the left. Remaining physical exam was within normal limits.

IV-2's last 4 years were complicated by additional physical problems: agenesis of the corpus callosum identified on neonatal MRI; neonatal seizures controlled with phenobarbital; hearing deficits requiring bilateral hearing aids; gastrointestinal (GI) reflux and failure to thrive requiring only pureed food; numerous dental caries necessitating oral surgery for dental restorations and extractions. Ophthalmologic examination documented normal vision without structural defects. With respect to developmental delays, IV-2 sat unassisted at 12 months; is making efforts to crawl at age 4 but walked with a walker at age 3; can vocalize but does not have intelligible words; has limited knowledge of ASL and through this, expresses his wishes. Family members report inability to follow simple commands.

III-1's parents had eight pregnancies: five ended in first trimester miscarriage, three resulted in liveborn, female offspring; III-1, III-2, and III-3. Due to III-1's balanced translocation, III-2 and III-3 underwent peripheral blood karyotype analysis. III- 2 was found to carry a balanced translocation 46,XX,t $(4 ; 18)(\mathrm{p} 12 ; \mathrm{p} 11.2)$ (band resolution unavailable). III-3's karyotype is 46,XX. III-1's mother had six healthy siblings who all had healthy children. III-1's maternal grandparents reportedly had at least six miscarriages. Further details regarding karyotype analysis of extended family members are unknown.

The frequency of reciprocal translocations in the general population is estimated at 1/500 (Gardner and Sutherland, 2004); 70\% of balanced reciprocal translocations are inherited. The likelihood of various pregnancy outcomes can be estimated based on chromosomes involved, gender of carrier parent, size of translocated segments, and family's reproductive history. Miscarriage rates approximate $50 \%$ in female patients such as III-2 and III-1, who carry balanced translocations and have a significant family history of recurrent miscarriage. In this family, the likelihood of a carrier female having a liveborn offspring with an unbalanced rearrangement is 20-25\% (Gardner and Sutherland, 2004). The chance of a liveborn unaffected neonate for carrier females nears $25-30 \%$. Options for future pregnancies include pre-implantation genetic diagnosis, prenatal diagnosis by CVS or amniocentesis, or ultrasound surveillance for abnormalities.

The translocation present in this family resulted in monosomy $18 \mathrm{p}$ and trisomy $4 \mathrm{p}$ in both IV-2 and III2's abortus. Monosomy $18 \mathrm{p}$ and trisomy $4 \mathrm{p}$ have both been described previously (Gonzalez et al., 1977). To our knowledge, this case report is the first to describe the combination of monosomy $18 p$ and $4 p$ concurrently in the same individual and the importance of noting the variable presentations associated with this combination. Monosomy $18 \mathrm{p}$, also known as deletion $18 \mathrm{p}$ syndrome, has been associated with a constellation of 


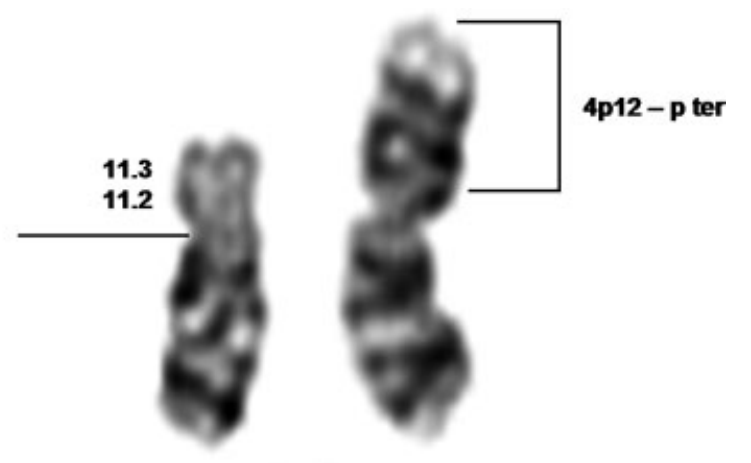

46,XX,der(18)t(4;18)(p12;p11.2)mat; band resolution 400

Figure 1-Individual IV-3: G banded partial karyotype of chromosome 18 demonstrating $18 \mathrm{p} 11.2$ breakpoint with deletion to $18 \mathrm{p}$ terminus, suggesting TGIF deletion due to the absence of $18 \mathrm{p} 11.3$

characteristic features including growth deficiency, as well as central nervous system, facial, teeth, and limb abnormalities (Jones, 2006). Average IQ of 45-50 has been reported. Many affected children are moderately to severely mentally retarded and experience language delays (Schinzel, 2001). Reported facial characteristics include ptosis, epicanthal folds, hypertelorism, and micrognathia; $29 \%$ of the affected individuals have dental caries. Uchida et al. (1965) reported on a family with $18 \mathrm{p}$ deletion syndrome in which one affected neonate was cebocephalic. Overhauser et al. (1995) described six individuals with holoprosencephaly and $18 \mathrm{p}$ deletions, suggesting that a critical region for holoprosencephaly maps to the $18 \mathrm{p} 11.3$ region, the locus for TG-interacting factor (TGIF) Figure 1 demonstrates a partial karyotype of chromosome 18 , with the p11.3 region deleted and replaced by $4 \mathrm{p}$. This is consistent with Overhauser's conclusion that the critical region for holoprosencephaly maps to $18 \mathrm{p} 11.3$ region, the locus for TGIF. TGIF mutations have been associated with non-syndromic holoprosencephaly. Testing for such mutations is now available clinically. The incidence of holoprosencephaly with an 18p deletion is estimated at 10\% (Schinzel, 2001). Other associations with $18 \mathrm{p}$ deletions, many clearly recognizable on prenatal ultrasound and immediately at birth have been reported, including genital anomalies, talipes equinovarus, cardiac defects, syndactyly, and fifth finger clinodacyly. However, monosomy 18p may go unrecognized at birth with facial, skeletal, and gonadal dysmorphology developing later (Schinzel, 2001).

Trisomy $4 \mathrm{p}$ has been associated with multiple phenotypic characteristics: cranial findings (microcephaly, hypertelorism, depressed nasal bridge with bulbous nasal tip, abnormal ear shape and position, and short neck); $15 \%$ incidence of associated congenital heart disease (Patel et al., 1995); anatomic GI abnormalities and feeding problems; and male genitalia abnormalities (micropenis, cryptorchidism, hypospadias, and hypoplastic scrotum). Of the affected individuals, $100 \%$ have single or multiple skeletal anomalies and psychomotor retardation. Most affected individuals exhibit postnatal growth delays. Karmous-Benailly et al. (2005) provide what is thought to be the first report of a fetus with trisomy $4 p$ and holoprosencephaly. When discussing classic trisomy $4 \mathrm{p}$ syndrome, the variability of clinical manifestations and phenotypic and neurodevelopmental outcomes remains a key point of discussion.

IV-2 and III-2's abortus, from the same family, exhibited extremely discordant phenotypic expression in the presence of the same unbalanced chromosome translocation. IV-2 is surviving with multiple physical and developmental problems including phenotypic and developmental characteristics of both monosomy 18p syndrome and trisomy $4 \mathrm{p}$ syndrome, but does not fit in either syndrome completely. III-2's abortus had more severe manifestations, presence of a proboscis indicating alobar holoprosencephaly, a finding consistent with previous reports of the association between monosomy 18p and holoprosencephaly. The profoundly different phenotypic presentations of the affected individuals observed in this family underline the difficulty in genetic counseling of patients regarding possible outcomes associated with particular unbalanced chromosome rearrangements, even when the affected individuals' rearrangement is the same. It also underscores the value of first trimester ultrasound that can facilitate identification and early detection of a lethal anomaly, allowing for early prenatal diagnosis and/or consideration of voluntary termination of pregnancy.

\section{REFERENCES}

Gardner RJM, Sutherland GR. 2004. Chromosome Abnormalities and Genetic Counseling (3rd edn). Oxford University Press: New York; 344-577.

Gonzalez CH, Sommer A, Meisner LF, Elejalde BR, Opitz JR. 1977. The trisomy $4 \mathrm{p}$ Síndrome: Case report and review. Am J Med Genet 1: $137-156$.

Jones KL. 2006. Smith's Recognizable Patterns of Human Malformation (6th edn). Elesevier: Philadephlia, PA; 954.

Karmous-Benailly H, Tabet AC, Thaly A, et al. 2005. Prenatal diagnosis of trisomy 4p: a new locus for holoprosencephaly? Prenat Diagn 25: 193-197.

Overhauser J, Mitchell HF, Zackai EH, Tick DB, Rojas K, Muenke M. 1995. Physical Mapping of the Holoprosencephaly Critical Region in18p11.3. Am J Med Genet 57: 1080-1085.

Patel SV, Dagnew H, Parekh AJ, et al. 1995. Clinical manifestations of trisomy 4p syndrome. Eur J Pediatr 154: 425-431.

Schinzel A. 2001. Catalogue of Unbalanced Chromosome Aberrations in Man (2nd edn). DeGruyter: Berlin; 966.

Uchida IA, McRae KN, Wang HC, Ray M. 1965. Familial short arm deficiency of chromosome 18 concomitant with arhinencephaly and alopecia congenital. Am J Hum Genet 17: 410-415. 